\title{
THE EFFECTS OF BOVINE SEMINAL PLASMA ON THE IMPEDANCE CHANGE FREQUENCY AND GLYCOLYSIS OF BOVINE EPIDIDYMAL SPERMATOZOA
}

\author{
H. M. DOTT \\ A.R.C. Unit of Reproductive Physiology and Biochemistry, Cambridge*
}

(Received 26th September 1973)

\begin{abstract}
Summary. Bovine seminal plasma was separated into a number of fractions by dialysis, ethanol extraction or gel chromatography. Bovine spermatozoa suspended in the non-dialysable fraction or the ethanolinsoluble fraction or in fractions either excluded from, or retained by, the Sephadex gel, all reduced the impedance change frequency and viability of the spermatozoa. The fractions separated by gel chromatography also caused the spermatozoa to use more glucose and rendered them eosinophilic sooner than spermatozoa suspended in Ringer or in fresh whole seminal plasma.
\end{abstract}

\section{INTRODUCTION}

Bovine seminal plasma affects bovine spermatozoa in a number of ways: bull spermatozoa from the epididymis have a lower respiratory rate than ejaculated spermatozoa (Lardy, Ghosh \& Plaut, 1949; Salisbury \& Graves, 1963; Salisbury, Graves, Nakabayashi \& Cragle, 1963); spermatozoa from the epididymis of the bull appear to lose some of their capacity to resist cold shock when they are ejaculated (Lasley \& Mayer, 1944; Bialy \& Smith, 1959); spermatozoa from the epididymis survive longer than ejaculated spermatozoa in vitro (Dott, 1958). These effects are produced by whole seminal plasma but whether any one effect is due to a single component of the mixture is not known. Lardy \& Ghosh (1952) attributed the decreased efficiency of the metabolism of ejaculated spermatozoa in part to cystine and glutathione, and Shannon (1962, 1965a, b) found that when seminal plasma was used to dilute bull semen, the sperm viability was reduced, an effect which he attributed to some substance with a sulphydryl-binding capacity. Bennett \& Dott (1966) found that the impedance change frequency (ICF) of epididymal spermatozoa was reduced when they were suspended in the fraction of the seminal plasma which contains most of the protein and their viability was similar to that of ejaculated spermatozoa.

The experiments described below are an extension of the study by Bennett \& Dott (1966) and were designed to discover the effect on the ICF and glycolysis of bovine epididymal spermatozoa of suspending them in bovine seminal plasma which had been fractionated in various ways.

\footnotetext{
* Postal address: Animal Research Station, 307 Huntingdon Road, Cambridge CB3 0JQ.
} 


\section{MATERIALS AND METHODS}

Spermatozoa

All the spermatozoa were obtained by aspiration from the epididymides of bulls after slaughter (Dott \& Skinner, 1967). Most of the bulls were Friesians (5 to 6 years old) whose progeny had failed to reach the standards required by the ARC or MMB progeny-testing schemes.

\section{Seminal plasma}

The seminal plasma was obtained from semen collected from Friesian bulls. Immediately after collection, the semen was centrifuged at $5000 \mathrm{~g}$ for $30 \mathrm{~min}$ and the supernatant was removed. The supernatant was free of spermatozoa and cytoplasmic droplets but not of submicroscopic particles (Dott \& Dingle, 1968).

The methods used to fractionate the seminal plasma were (1) dialysis, (2) ethanol extraction, and (3) gel chromatography.

Dialysis. A rapid dialysis method was used, the seminal plasma being dialysed against water and the non-dialysable portion brought back to the original volume by freeze-drying and resuspension in iso-osmotic saline. Two fractions were obtained: (a) non-dialysable seminal plasma (NDSP) which would not pass through the cellophane membrane, and (b) dialysable seminal plasma (DSP) which would pass through the cellophane membrane.

Ethanol extraction. Seminal plasma $(10 \mathrm{ml})$ was cooled to $0^{\circ} \mathrm{C}$ and shaken with $50 \mathrm{ml}$ pre-cooled $\left(-15^{\circ} \mathrm{C}\right) 80 \%$ ethanol at $0^{\circ} \mathrm{C}$. The suspension was centrifuged, the supernatant removed and the precipitate resuspended in a further $50 \mathrm{ml}$ of $80 \%$ ethanol. After centrifugation, the supernatant was discarded and the precipitate resuspended in water and freeze-dried. The powder was redissolved in a solution containing $110 \mathrm{~mm}-\mathrm{NaCl}, 40 \mathrm{~mm}-\mathrm{KCl}$ and $6.4 \mathrm{~mm}$-phosphate buffer $(\mathrm{pH} 7 \cdot 4)$. The suspension was dried on a rotary evaporator, and the residue was resuspended in $10 \cdot 0 \mathrm{ml} \mathrm{H}_{2} \mathrm{O}$.

Gel chromatography. The seminal plasma $(5.0 \mathrm{ml})$ was absorbed on a Sephadex G-50 column in 10 mm-phosphate buffer ( $\mathrm{pH} \mathrm{7.1).} \mathrm{The} \mathrm{column} \mathrm{had} \mathrm{a} \mathrm{total}$ volume of approximately $100 \mathrm{ml}$ and a void volume (to Dextran 2000) of $45 \mathrm{ml}$. The flow rate was $36 \mathrm{ml} / \mathrm{hr}$ and 100 fractions each of $2.4 \mathrm{ml}$ were collected. Absorption at $280 \mathrm{~nm}$ was measured. There were three groups of collection tubes in which absorption was high (Text-fig. 1). The liquid in each tube of a group was pooled with the liquid from the other tubes of the group and the volume measured. The volume was reduced by freeze-drying and an appropriate volume of water and phosphate buffer was added to bring the volume to $5.0 \mathrm{ml}$, and to adjust the osmotic pressure to the level in the original seminal plasma at a $\mathrm{pH}$ of $7 \cdot 1$. Three fluid fractions were obtained which were labelled $\mathrm{Pl}$ (high mol. wt), P2 (medium mol. wt) and P3 (low mol. wt). The peak due to P1 coincided with the place at which Dextran 2000 was released from the column. Fructose was associated with $\mathrm{P} 3$ but not with the other two fractions. No attempt was made to investigate the chemical composition of $\mathrm{P} 1, \mathrm{P} 2$ or P3.

It is interesting that the spectrum of absorption for P1 and the solution of material precipitated by ethanol are very similar (Text-fig. 2). 


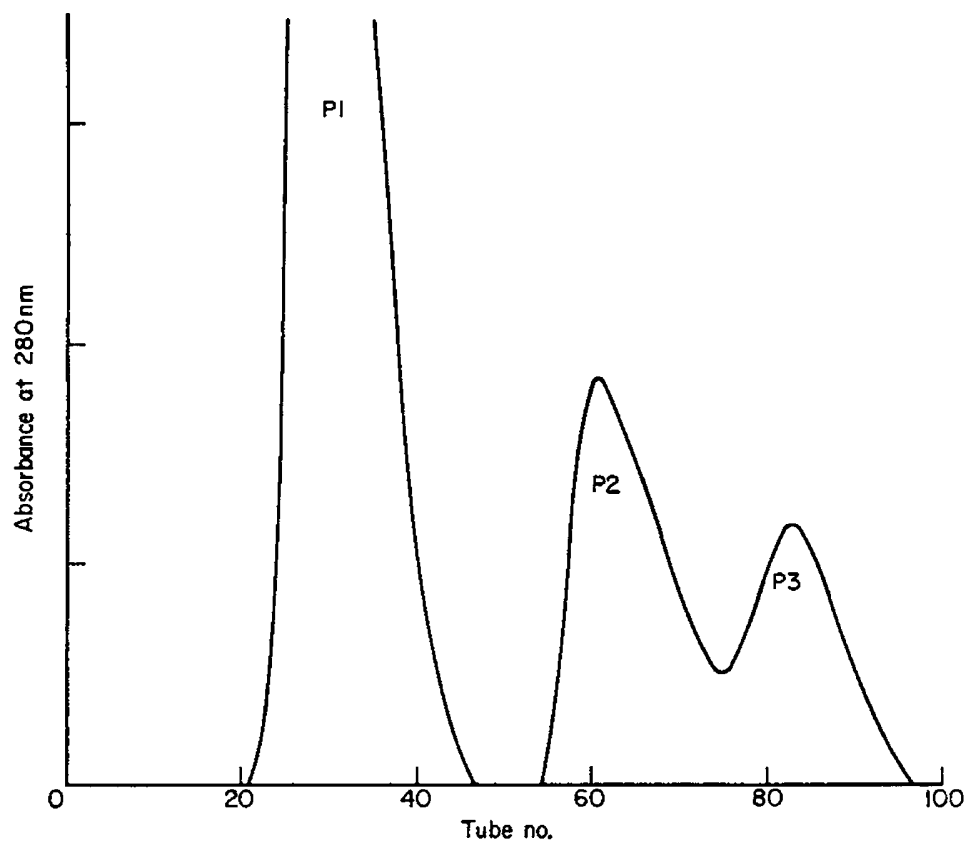

Text-rig. 1. Absorbance of the fraction of bovine seminal plasma recovered from a Sephadex G-50 column run at $15 \mathrm{ml} / \mathrm{hr}$. Fraction size: $2 \cdot 4 \mathrm{ml}$ in $10 \mathrm{~mm}$-phosphate buffer at $4^{\circ} \mathrm{C}$.



Text-pig. 2. Absorption spectrum between 250 and $350 \mathrm{~nm}$ of the fractions of bovine seminal plasma precipitated by ethanol (upper curve) and the Fraction Pl recovered from Sephadex G-50 (lower curve). 
Assessment of ICF and viability

The ICF of samples was estimated in the continuous flow dialysis apparatus (CFDA) as described by Dott \& Walton (1960). In all experiments dialysis continued for $24 \mathrm{hr}$.

Since Bennett \& Dott (1966) had shown that the ICF of undiluted epididymal spermatozoa did not differ from epididymal spermatozoa diluted with KrebsRinger, undiluted controls were not included in the experiment described here. The degree of dilution throughout was one drop of epididymal spermatozoa to seven drops of test solution, which brought the sperm density to approximately the same level as that found in ejaculated semen.

The results of experiments in the CFDA were transformed to logs and assessed by analysis of variance (Dott \& White, 1964).

\section{Metabolism}

Epididymal semen was divided into four aliquots which were diluted with (i) seminal plasma, (ii) $\mathrm{P} 1$, (iii) $\mathrm{P} 2$ plus $\mathrm{P} 3$, (iv) $2.9 \%$ citrate. This dilution reduced the sperm density to between $750 \times 10^{6}$ and $1800 \times 10^{6}$ spermatozoa $/ \mathrm{ml}$. Glucose was added to produce a concentration of $10 \mathrm{~mm}$-glucose in the final mixture.

The suspensions were incubated under nitrogen in Thunberg tubes for $2 \mathrm{hr}$ at $37^{\circ} \mathrm{C}$. Duplicate samples were removed every $30 \mathrm{~min}$ for glucose determination and at the beginning and end of the period of incubation for lactate determinations.

Samples were stained with nigrosin-eosin to determine the live/dead sperm ratio at the beginning and end of incubation and the sperm density was estimated with a Spekker absorptiometer at the end of incubation.

The manual method described by Morley, Dawson \& Marks (1968) was used to determine the concentrations of glucose.

The concentration of lactate was determined enzymatically, using the method recommended by the Boehringer Corporation (London) Ltd.

Glucose and lactate concentrations were expressed as $\mathrm{mg} / 10^{9}$ 'live' spermatozoa. From these findings, the fractional decline in sperm glycolytic activity per minute $(\mathrm{K})$ and the maximum rate of glucose uptake (MGU) were calculated (Mixner, Mather \& Freund, 1957) as well as the quantity of glucose used by $10^{9}$ 'live' spermatozoa in four successive periods of $30 \mathrm{~min}$. The latter figures were assessed by analysis of variance.

\section{RESULTS}

ICF and viability

(a) Dilution of spermatozoa from the epididymis with seminal plasma, NDSP, DSP and an ethanol insoluble fraction of seminal plasma. The results of seven experiments are illustrated in Text-fig. 3. All the diluents containing seminal plasma or portions of seminal plasma reduced the ICF compared with the aliquot diluted with Krebs-Ringer bicarbonate. The effect was observed within $1 \mathrm{hr}$ from the beginning of dialysis. Dilution with DSP had less effect than dilution with the other three diluents. 
(b) Dilution with fraction of seminal plasma separated by gel chromatography. The results of three experiments are illustrated in Text-fig. 4. Fractions P1 and P3 both reduced the ICF to the same extent and in the same time as whole seminal plasma, but samples diluted with P2 did not differ from the controls.

\section{Glucose uptake and lactate production}

(a) Glycolysis in the presence of fructose. Whole seminal plasma, DSP and P3 all contain fructose and it has been reported that glycolysis is lower in the presence of fructose than in its absence (ejaculated bull spermatozoa-van Tienhoven,

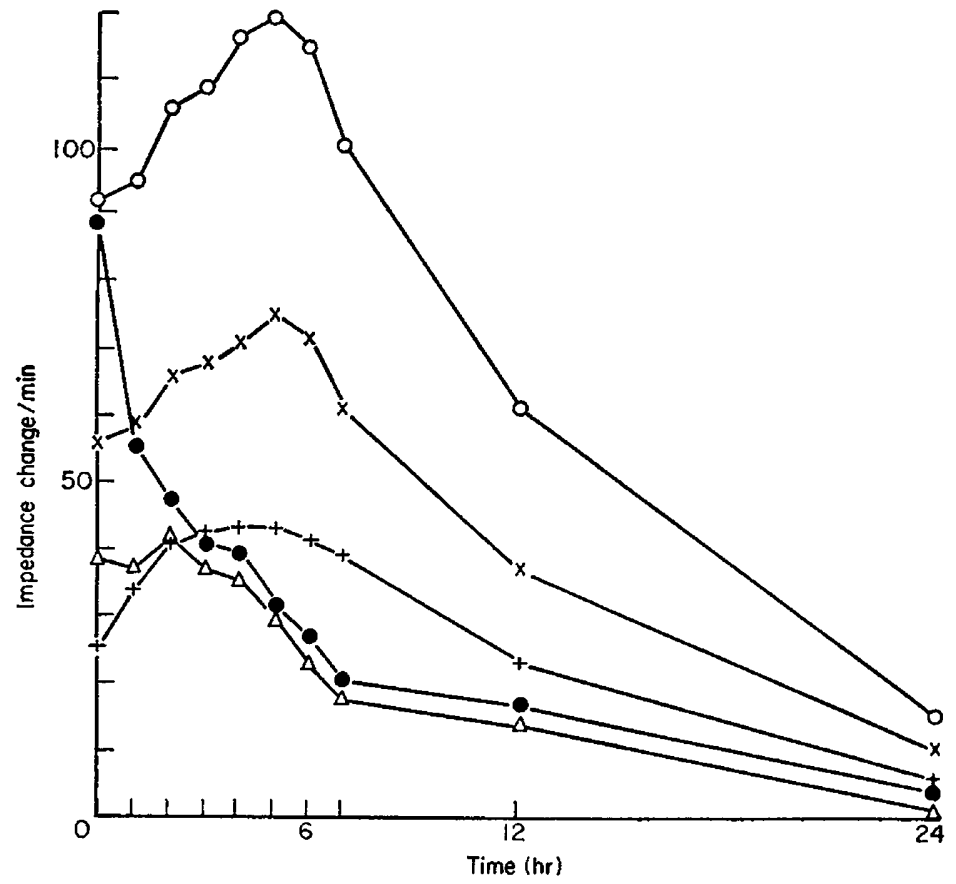

TEXT-FIG. 3. Impedance change frequency of bovine epididymal spermatozoa in the continuous flow dialysis apparatus during dialysis for $24 \mathrm{hr}$ under aerobic conditions with fructose included in the Krebs-Ringer used as a dialysis fluid. The spermatozoa within the dialysis bag were suspended in: $O$, Ringer; + , ethanol-insoluble portion of seminal plasma; non-dialysable seminal plasma; $\Delta$, fresh seminal plasma; $x$, dialysable seminal plasma.

Salisbury, VanDemark \& Hansen, 1952; washed fowl spermatozoa-van Tienhoven, 1960; washed, ejaculated ram spermatozoa-Mann, 1951). If fructose lowers the glycolysis of bull spermatozoa from the epididymis, the rate of glycolysis in fractions containing fructose would be lower than in fractions which did not contain fructose. Bull epididymal spermatozoa were incubated with $10 \mathrm{~mm}$-glucose in the presence of 40,30, 20,10 and $5 \mathrm{~mm}$-fructose. The total hexose concentration was maintained at $50 \mathrm{~mm}$ by including an appropriate concentration of galactose in place of the fructose. The rate of glycolysis was not affected by the concentration of fructose (Table 1).

(b) Glycolysis in seminal plasma and fractions of seminal plasma. The spermatozoa 


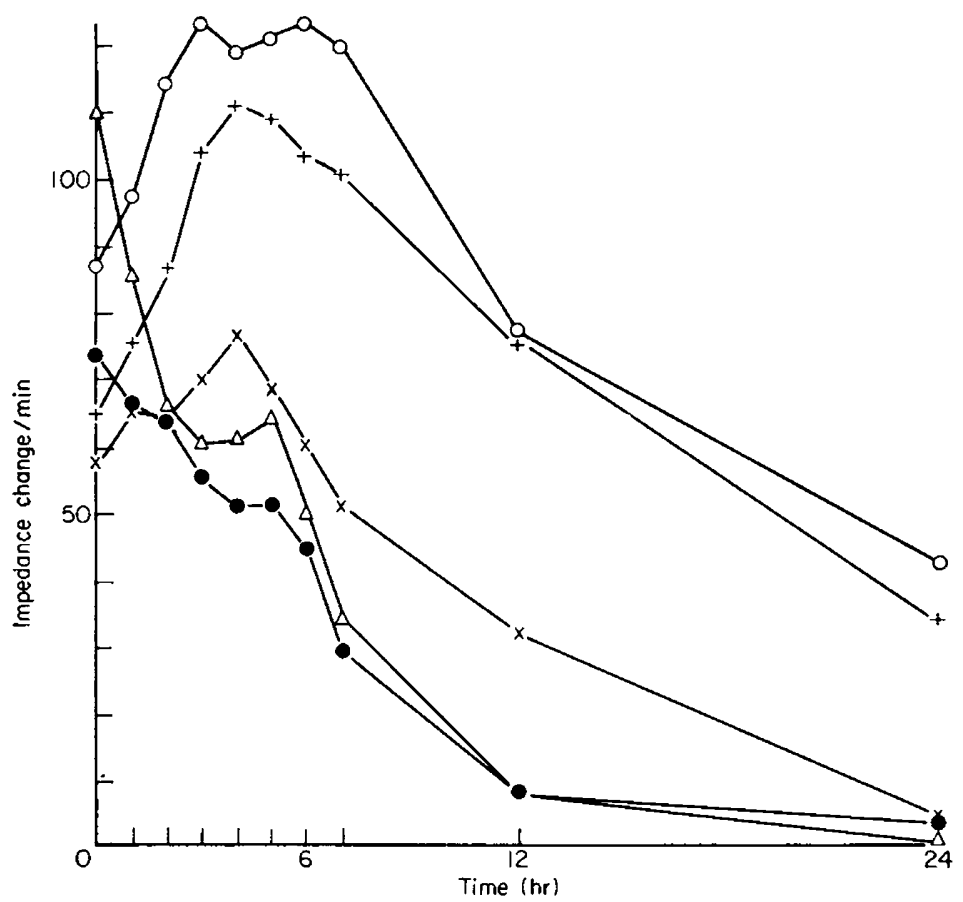

Text-Fig. 4. Impedance change frequency of bovine epididymal spermatozoa in the continuous flow dialysis apparatus during dialysis for $24 \mathrm{hr}$ under aerobic conditions with fructose included in the Krebs-Ringer used as a dialysis fluid. The spermatozoa within the dialysis bag were suspended in: $O$, Ringer; +, Fraction P2; $\odot$, Fraction P1; $x$, Fraction $P 3 ; \Delta$, fresh seminal plasma.

from seven epididymides used in these experiments were suspended in $\mathrm{Pl}$, a mixture of P2 and P3, seminal plasma or citrate buffer. The glucose used by $10^{9}$ 'live' spermatozoa in $2 \mathrm{hr}$ is shown in Table 2. The spermatozoa in citrate and seminal plasma used least glucose.

Table 1. The effect of fructose concentration on the amount of glucose used by $10^{9}$ 'live' bovine epididymal spermatozoa under nitrogen in successive periods of $30 \mathrm{~min}$ at $37^{\circ} \mathrm{C}$

\begin{tabular}{c|c|c|c|c|c|c|c}
\hline \multirow{2}{*}{$\begin{array}{c}\text { Time } \\
(\mathrm{hr})\end{array}$} & \multicolumn{7}{c}{ Fructose concentration $(\mathrm{mM})$} \\
\cline { 2 - 7 } & 40 & 30 & 20 & 10 & 5 & 0 & Mean \\
\hline 0 to $\frac{1}{2}$ & 0.9547 & 1.0287 & 0.9467 & 0.7752 & 0.8506 & 0.6457 & 0.8656 \\
$\frac{1}{2}$ to 1 & 0.8072 & 0.8224 & 0.8840 & 0.8783 & 0.8024 & 0.8334 & 0.8413 \\
1 to $1 \frac{1}{2}$ & 0.8346 & 0.6796 & 0.8378 & 0.8044 & 0.7367 & 0.9718 & 0.8108 \\
$1 \frac{1}{2}$ to 2 & 1.0505 & 0.9276 & 0.5938 & 0.5480 & 0.8875 & 1.1815 & 0.8732 \\
Miean 30 min & 0.9117 & 0.8646 & 0.8156 & 0.7639 & 0.8144 & 0.9131 & 0.8472 \\
\hline
\end{tabular}

Each glucose value ( $\mathrm{mg}$ ) is the mean of five experiments. Neither the differences between fructose concentrations nor those between 30 -min incubation periods were significant. 
The rate of decline of glucose uptake $(\mathbf{K})$ was fairly constant in these two diluents and was greater than the rate of decline in P1 and P2 plus P3 (Table 3). The calculated maximum glucose uptake (i.e. the amount of glucose used in the first minute of incubation) is not very different in the four groups (Table 3).

Table 2. Glucose consumption and lactate production by bovine epididymal spermatozoa and the change in the proportion of noneosinophilic ('live') spermatozoa during a 2-hr incubation under anaerobic conditions at $37^{\circ} \mathrm{C}$

\begin{tabular}{l|c|c|c|c}
\hline & $\begin{array}{c}\text { Glucose } \\
\text { used* }\end{array}$ & $\begin{array}{c}\text { Lactate } \\
\text { produced } \dagger\end{array}$ & $\begin{array}{c}\% \text { 'live' sperm. } \\
\text { before } \\
\text { incubation }\end{array}$ & $\begin{array}{c}\% \text { 'live' sperm. } \\
\text { after } \\
\text { incubation }\end{array}$ \\
\hline Citrate buffer & 1.628 & 1.422 & 86 & 85 \\
Fraction P1 & 2.308 & 2.419 & 62 & 30 \\
Fractions P2 plus P3 & 2.502 & $(4.189)$ & 65 & 44 \\
Fresh seminal plasma & 1.801 & 1.55 & 78 & 75 \\
\hline
\end{tabular}

Both glucose consumption and lactate production are expressed in $\mathrm{mg} / 10^{9}$ 'live' spermatozoa.

* Mean of seven experiments.

$\dagger$ Mean of six experiments.

Table 3. Decline in rate of glycolysis/minute $(\mathbf{K})$ and maximum rate of glucose uptake (MGU) of bovine epididymal spermatozoa incubated at $37^{\circ} \mathrm{C}$ under anaerobic conditions

\begin{tabular}{|c|c|c|c|c|c|}
\hline \multirow{2}{*}{ Diluent } & \multicolumn{4}{|c|}{$\begin{array}{c}\kappa \text { calculated from readings over various time } \\
\text { intervals }\end{array}$} & \multirow{2}{*}{$\begin{array}{l}M G U \text { in } m g \text { glucose } / 10^{9} \\
\text { live sperm./min at time } 0\end{array}$} \\
\hline & 0 to $1 \mathrm{hr}$ & $\frac{1}{2}$ to $1 \frac{1}{2} h r$ & 1 to $2 \mathrm{hr}$ & 0 to $2 \mathrm{hr}$ & \\
\hline $\begin{array}{l}\text { Gitrate buffer } \\
\text { Fraction P1 } \\
\text { Fractions P2 plus P3 } \\
\text { Seminal plasma }\end{array}$ & $\begin{array}{l}0.0113 \\
0.0099 \\
0.0054 \\
0.0123\end{array}$ & $\begin{array}{l}0.0121 \\
0.0033 \\
0.002 \\
0.0181\end{array}$ & $\begin{array}{l}0.0182 \\
0.0222 \\
0.002 \\
0.0134\end{array}$ & $\begin{array}{l}0.0131 \\
0.009 \\
0.0029 \\
0.0154\end{array}$ & $\begin{array}{l}0.0256 \\
0.031 \\
0.0246 \\
0.031\end{array}$ \\
\hline
\end{tabular}

$\mathrm{K}=0.0002 / \mathrm{min} . \mathrm{MGU}=0.0285 \mathrm{mg}$ glucose used by $10^{9}$ 'live' spermatozoa in the first minute of incubation.

The lactate production was measured in six of the seven experiments. Except for the P2 plus P3 group, the lactate produced was of the same order as the glucose used (Table 2). The figure for P2 plus P3 resulted from extraordinarily high estimates at the end of two experiments. In both these experiments, there was a massive increase in the percentage of stained ('dead') spermatozoa during incubation (from approx. $20 \%$ to $80 \%$ ); as the lactate production was expressed in terms of $\mathrm{mg} / 10^{9}$ 'live' spermatozoa, the results may have been produced by faulty estimation and/or faulty assumptions in the calculations.

In making the calculation of lactate produced by $10^{9}$ 'live' spermatozoa, it was assumed that when there was an increase in the proportion of dead spermatozoa during incubation this increase was linear with respect to time. If most 
of the spermatozoa produced lactate during the entire period but died just before the end of incubation, then the correction made would lead to a spuriously high figure for lactate production $/ 10^{9}$ 'live' spermatozoa.

There was no increase in the proportion of eosinophilic spermatozoa when citrate buffer or seminal plasma was used as the suspending medium (Table 2) but, when P1 or P2 plus P3 was used, the proportion of dead spermatozoa was higher initially and increased during incubation.

\section{DISCUSSION}

The results of the experiments in the continuous flow dialysis apparatus confirm the results presented by Bennett \& Dott (1966) that suspension of bull epididymal spermatozoa in bull seminal plasma is followed by an immediate reduction of the impedance change frequency (ICF) and a reduction in the length of time during which the ICF can be detected (Text-fig. 3). The observations have been extended to show that the effect on ICF and viability is brought about by various fractions of the seminal plasma. In a study of glycolysis, it was shown that whole seminal plasma did not affect the glycolytic efficiency of the spermatozoa but that two of the components of seminal plasma which reduced the ICF also reduced the glycolytic efficiency and rendered a higher proportion of the spermatozoa eosinophilic.

Unlike ICF, which is probably a group phenomenon, glycolysis can be related to an individual spermatozoon and it is therefore best expressed in terms of the number of active cells. The problem is to decide what an active cell is in this context. For the purpose of the calculations, it was decided to equate eosinophilia in a spermatozoon with absence of glycolytic activity. There are two factors which support this: (a) eosinophilia among spermatozoa is found in association with some morphological changes which appear to be irreversible and which are not seen in motile spermatozoa, and (b) the proportion of eosinophilic spermatozoa increases when the population is subjected to conditions which render a high proportion of spermatozoa irreversibly immotile (e.g. cold shock-Hancock, 1951; Wales \& White, 1959; Dott, 1968). This indirect assessment of the proportion of motile spermatozoa may not be absolutely correct but it is probably justifiable to use it to compare the metabolism of two populations of spermatozoa.

The arguments for the mathematical treatment of the glucose estimations have been presented by Mixner et al. (1957) in a paper on fructolysis. Two parameters are determined: the maximum rate of glucose use (MGU) and the rate of decline in glycolytic activity (glycolysis coefficient, $\mathrm{K}$ ). These two parameters can be used to calculate the amount of glucose used in a given time ( $\mathrm{mg}$ glucose by $10^{9}$ spermatozoa in $1 \mathrm{hr}$ at $37^{\circ} \mathrm{C}$-the glycolysis index). The validity of the mathematical treatment can be tested by comparing the glycolysis index obtained by measurement with that calculated from values of $K$ and MGU. It will be seen from the figures for the first hour given in Table 4 that the agreement is very close. The advantages of using this method on the data in this experiment are that any differences found between treatments can be attributed to the particular parameter or parameters responsible. The sperma- 
tozoa suspended in P1 or P2 plus P3 used more glucose than those suspended in citrate buffer and seminal plasma (Tables 2 and 4 ) because the rate of decline of reaction $(\mathrm{K})$ was lower and not because the reaction was faster at the beginning of incubation (Table 2). The $\mathrm{K}$ value remained lower despite the decreasing proportion of spermatozoa that were non-eosinophilic. This would suggest that P1 and P2 plus P3 were having at least two actions on the spermatozoa, (1) causing them to use more glucose and (2) rendering them eosinophilic sooner than if they had been in buffer. The effect on staining has been confirmed in a more extensive series of experiments (H. M. Dott, unpublished observations).

The purpose of this work has been achieved but only to a limited extent because the amount of material obtainable from each bull is small and only a few suitable bulls were readily available. Attempts to obtain suitable spermatozoa by creating a fistula in a bull (Bennett $\&$ Rowson, 1963) have failed and it has not been possible to reverse the effect of seminal plasma by washing ejaculated spermatozoa and suspending them in another fluid. The epididymal-like cells described by Salisbury \& Graves (1963) are not suitable for this type of experiment.

Table 4. Glycolysis index of bovine spermatozoa incubated at $37^{\circ} \mathrm{C}$ under anaerobic conditions for $\mathrm{l} \mathrm{hr}$

\begin{tabular}{l|c|c|c|c}
\hline & Buffer & Fraction P1 & Fractions P2 plus P3 & $\begin{array}{c}\text { Seminal } \\
\text { plasma }\end{array}$ \\
\hline $\begin{array}{c}\text { Calculated } \\
\text { from K and MGU }\end{array}$ & 1.08 & 1.46 & 1.38 & 1.23 \\
$\begin{array}{c}\text { Addition of } \\
\text { measured values }\end{array}$ & 1.117 & 1.46 & 1.36 & 1.29 \\
\hline
\end{tabular}

Because the material for testing the components of seminal plasma was so scarce (i.e. epididymal spermatozoa), it was not feasible to test each component separately and relatively crude methods of separation were used in these experiments. The possible importance of this effect in the field of preservation of bull spermatozoa warrants a more detailed study of the components of seminal plasma so that the active factor (or factors) can be identified and their activity inhibited. A similar study of ram semen might prove even more rewarding since Dott (1961) has shown that incubation of ram spermatozoa with seminal plasma reduces the conception rate obtained at subsequent artificial insemination.

\section{ACKNOWLEDGMENTS}

I wish to thank Dr R. A. P. Harrison for advice on some of the methods, $\mathrm{Mr}$ G. F. Foster for technical assistance and Professor T. R. R. Mann for his interest and help in the preparation of the manuscript.

\section{REFERENGES}

Bennett, J. P. \& Dotr, H. M. (1966) An effect of bovine seminal plasma on the impedance change frequency of epididymal spermatozoa collected from the living bull. F. Reprod. Fert. 12, 327. 
Bennett, J. P. \& Rowson, L. E. A. (1963) A fistula for the collection of epididymal semen from the bull. F. Reprod. Fert. 6, 61 .

Bialy, G. \& SMith, V. R. (1959) Gold shock of epididymal spermatozoa. F. Dairy Sci. 42, 2002.

Dotr, H. M. (1958) Species differences in the metabolism of epididymal spermatozoa. Stud. Fert., Oxford, 10, 73.

Dort, H. M. (1961) The fertility of ram semen treated in the continuous flow dialysis apparatus. 4th Int. Congr. Anim. Reprod., The Hague, p. 873.

Dotr, H. M. (1968) Effect of sudden cooling on the proportion of eosinophilic bull, ram and rabbit ejaculated spermatozoa in diluted and undiluted semen.6 6th Int. Congr. Anim. Reprod. \& A.I., Paris, 2, 1235.

DotT, H. M. \& Dingle, J. T. (1968) Distribution of lysosomal enzymes in the spermatozoa and cytoplasmic droplets of bull and ram. Expl Cell Res. 52, 523.

Dorr, H. M. \& SkinNer, J. D. (1967) A re-assessment of extra gonadal sperm reserves in Suffolk rams. 7. agric. Sci., Camb. 69, 293.

DotT, H. M. \& WaLton, A. (1960) Effects of dilution and washing on ram spermatozoa studied by the flow dialysis technique. F. Reprod. Fert. 1, 350.

DotT, H. M. \& WhITE, I. G. (1964) Effect of potassium on ram spermatozoa studied by a flow dialysis technique. F. Reprod. Fert. 7, 127.

Hancock, J. L. (1951) A staining technique for the study of temperature shock in semen. Nature, Lond. $167,323$.

LARDY, H. A. \& GHosh, D. (1952) Comparative metabolic behavior of epididymal and ejaculated mammalian spermatozoa. Ann. N.Y. Acad. Sci. 55, 594.

Lardy, H. A., Ghosh, D. \& PlaUt, E. W. E. (1949) A metabolic regulator in mammalian spermatozoa. Science, N.Y. 109, 365.

LASLEY, J. F. \& MAYER, D. T. (1944) A variable physiological factor necessary for the survival of bull spermatozoa. F. Anim. Sci. 3, 129.

Mann, T. R. R. (1951) Mammalian semen: composition, metabolism and survival. Biochem. Soc. Symp. 7, 11.

Mrxner, J. P., Mather, R. E. \& Freund, M. (1957) Bovine semen metabolism. 1. Methods for expressing fructolytic activity. F. Dairy Sci. 40, 142.

Morley, G., Dawson, A. \& Marks, V. (1968) Manual and Autoanalyzer methods for measuring glucose using guaiacum and glucose oxidase. Proc. Ass. clin. Biochemists, 5, 42.

Salisbury, G. W. \& Graves, G. N. (1963) Substrate-free epididymal-like bovine spermatozoa. $\mathcal{F}$. Reprod. Fert. 6, 351.

Salisbury, G. W., Graves, G. N., Nakabayashi, N. T. \& Gragle, R. G. (1963) Observations on the ascorbic metabolism of bull and goat epididymal spermatozoa. 7. Reprod. Fert. 6, 341.

Shannon, P. (1962) Effect of additions of volatile fatty acids on the viability and fertility of diluted bovine semen. Nature, Lond. 196, 1225.

Shannon, P. (1965a) Contribution of seminal plasma, sperm numbers, and gas phase to dilution effects of bovine spermatozoa. F. Dairy Sci. 48, 1357.

Shannon, P. (1965b) Presence of a heat labile toxic protein in bovine seminal plasma. J. Dairy Sci.48, 1362.

van Tienhoven, A. (1960) The metabolism of fowl sperm in different diluents. F. agric. Sci., Camb. $54,67$.

van Tienhoven, A., Salisbury, G. W., VanDemark, N. L. \& Hansen, R. G. (1952) The preferential utilization by bull spermatozoa of glucose as compared to fructose. F. Dairy Sci. 35, 637

WALEs, R. G. \& WhITE, I. G. (1959) The susceptibility of spermatozoa to temperature shock. F. Endocr. $19,211$. 